\title{
Optical Milli-Meter Wave Signal Generation using Frequency Octupling Without Optical Filter
}

\section{M.Baskaran, K.Vijayakumar}

\begin{abstract}
In this paper, we show a practical technique to produce a great octupledfrequency optical Millimeter-Wave (MMW) signal utilizing two Mach-Zehnder modulators associated in course. A Continuous Wave (CW) Laser, two LiNb MachZehnder Modulators(MZM) and a PIN Photodiode are utilized in the MMW signal age A careful change of Direct Current $(D C)$ inclination, tweak index(MI) and the presentation of a $90^{\circ}$ stage move between the 2 fell stages guarantees the age of recurrence octupled MMW signal.80 $\mathrm{GHz}$ RF signal is produced from a 10 $\mathrm{GHz} \mathrm{RF}$ Local Oscillator (LO) with a most extreme reachable with Optical Sideband Suppression Ratio (OSSR) of $57 \mathrm{~dB}$ and a Radio Frequency Spurious Suppression Ratio (RFSSR) of beyond what $42 \mathrm{~dB}$ can be gotten. An optical bearer is tweaked, and unadulterated \pm fourth-request sidebands are produced. The whole connection is reproduced utilizing Optisystem Software.
\end{abstract}

Keywords: Optical millimeter-wave, Mach-Zehnder Modulator, Frequency-Octupling, Radio over Fiber, Laser Diode.

\section{INTRODUCTION}

A critical increment in look into interests towards the optical age of recurrence tunable MMW signals is because of a lot of utilizations that it draws in. A couple of significant applications incorporate radar, therapeutic imaging, current instrumentation and remote correspondence frameworks. The matchless quality of MMW signal age in the optical space over the electrical area is because of two or three reasons .One explanation is that it underpins bigger data transfer capacity prerequisites and the other being enormous recurrence tunability. [1,3]. There have been different exhibits on creating optical MMW signals.Non direct impacts, for example, Stimulated Brullion Scattering have been abused in the age of MMW signals[4].The utilization of four wave blending in an exceptionally non straight Fiber(HNLF) and optical recurrence up-change method utilizing an outer modulator have additionally been suggested[5-7]. The optical recurrence up-change method stands best among these systems inferable from its trait of sound sideband generation[8]. This property guarantees the age of a stable MMW signal. A solitary MZM one-sided at least transmission indicate was utilized accomplish recurrence multiplying [9].An improvement in recurrence augmentation factor is seen in [10]. Recurrence quadrupling is acknowledged utilizing a solitary MZM one-sided at greatest transmission point to stifle all the odd request sidebands.A disadvantage of this methodology is that an optical channel is required for optical bearer concealment.
A filterless strategy for creating MMW signal guarantees diminished framework intricacy and improved recurrence tunabilty[2].A channel free technique for producing quadrupled optical MMW siganls utilizing 2 MZMs onesided at least transmission focuses is displayed in [11]. Microwave recurrence sextupling can be acknowledged with the assistance of two double terminal MZMs [12]. It can likewise be acknowledged utilizing a stage modulator and a MZM [13]. The utilization of stage modulator would create numerous optical sidebands that are not expected in this way they are not utilized in the vast majority of the applications. In [14] with the utilization of two MZMs, the various sounds are smothered and just the \pm third-request sidebands are gotten.

In this paper, we propose a straightforward strategy to produce a top notch octupled recurrence optical MMW utilizing two fell MZMs. The RF driving sign is presented with a $90^{\circ}$ stage move between the two fell stages. Two $180^{\circ}$ stage shifters are utilized to acquaint a $180^{\circ}$ stage move with the info microwave signal that is applied to one of the anodes of every one of the MZMs. The CW laser is the optical source and the RF signal is genearated by the microwave signal generator. At the yield of the first MZM all the odd sounds get stifled and just the second fourth request sidebands alongside the bearer are produced. Also, just the \pm fourth-request sidebands with the optical transporter being smothered exists at the yield of the second MZM. Also, subsequently the recurrence octupled signal is created

\section{PRINCIPLE}

The schematic chart of the proposed plan is delineated in Figure 1.A sinusoidal microwave signal produced from a microwave signal generator is applied to the one arm of the first MZM and a similar sign with a stage move $180^{\circ}$ is applied to the next arm of the equivalent MZM.The \pm 2 ndrequest sidebands with the optical bearer is gotten at the yield of the first MZM.

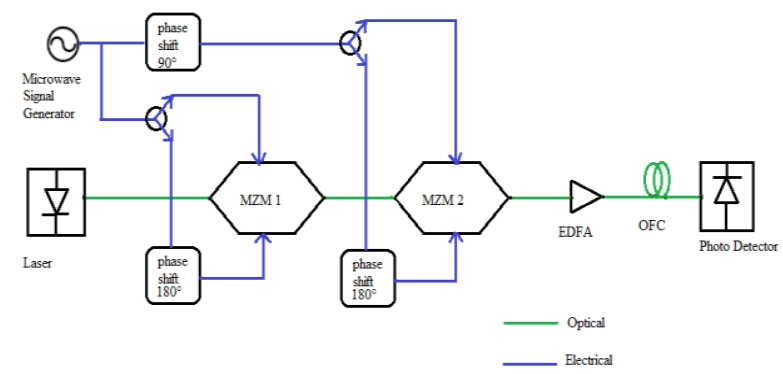

Figure 1.Schematic diagram of the proposed scheme

K. Vijayakumar, Associate Professor, St. Joseph's Institute of Technology, Chennai (Tamil Nadu), India

Baskaran.M, Associate Professor, Department of Electronics and Communication Engineering, Sri Venkateswaraa College of Technology, Vadakal (Tamil Nadu), India.

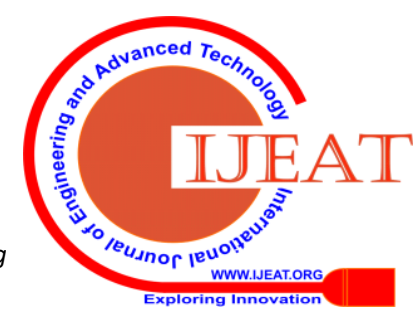


The CW laser articulation can given $\operatorname{asEc}(\mathrm{t})=\operatorname{Ecexp}(j c t)(1)$ where Ecis the abundancy of the optical field, and $c=2 \pi \mathrm{fc}$ is the rakish recurrence of the optical transporter. The RFdriven sign applied to the first MZM,

$v_{a}(t)=V_{R F} \sin \left(\omega_{R F} t\right) v_{b}(t)=V_{R F} \sin \left(\omega_{R F} t+\Delta \emptyset\right)$

Where the stage move $\Delta^{\varnothing}=180^{\circ}$ is presented between the RF driving sign on the arms of the MZM and VRFand RFare, individually, the sufficiency and the rakish recurrence of the microwave signal. The yield of the optical sign from the first MZM can be numerically communicated as

$$
\begin{gathered}
E_{01}(t)=\frac{\alpha}{2} E_{c} e^{j \omega_{c} t\left[e^{\left(\frac{j \pi v_{b}(t)}{v_{\pi R F}}\right)}\right.} \\
\left.+e^{\left(\frac{j \pi v_{a}(t)}{v_{\pi R F}}\right)}\right] \\
=\frac{\alpha}{2} E_{c} e^{j \omega_{c} t}\left[e^{\left(-\frac{j \pi V_{R F} \sin \left(\omega_{R F} t\right)}{v_{\pi R F}}\right)}\right. \\
\left.+e^{\left(j \pi V_{R F} \frac{\sin \left(\omega_{R F} t\right)}{v_{\pi R F}}\right)}\right] \\
=\frac{\alpha}{2} E_{c} e^{j \omega_{c} t}\left[e^{-j m \sin \left(\omega_{R F} t\right)}+\right. \\
\left.e^{j m \sin \left(\omega_{R F} t\right)}\right]
\end{gathered}
$$

By applying the Jaccobi-Anger expansion on Equation (3), $E_{01}(t)$ becomes,

$$
\begin{gathered}
\frac{\alpha}{2} E_{c} e^{j \omega_{c} t}\left[\sum_{n=-\infty}^{\infty} J_{n 1}(m) e^{j\left(n \omega_{R F} t+n \pi\right)}\right. \\
\left.+\sum_{n=-\infty}^{\infty} J_{n} e^{j\left(n \omega_{R F} t\right)}\right] \\
=\frac{\alpha}{2} E_{c} e^{j \omega_{c} t} \\
{\left[\sum_{n=-\infty}^{\infty} J_{n}(m) e^{j\left(n \omega_{R F} t+\frac{n \pi}{2}+\frac{n \pi}{2}\right)}\right.} \\
\left.+\sum_{n=-\infty}^{\infty} J_{n}(m) e^{j\left(n \omega_{R F} t+\frac{n \pi}{2}-\frac{n \pi}{2}\right)}\right] \\
=\frac{\alpha}{2} E_{c} e^{j \omega_{c} t}\left[\sum_{n=-\infty}^{\infty} J_{n}(m)\left(e^{j\left(\frac{n \pi}{2}\right)}+e^{-j\left(\frac{n \pi}{2}\right)}\right)\right] \\
=\alpha E_{c} J_{n}(m) \cos \left(\frac{n \pi}{2}\right) \\
\left.\sum_{n=-\infty}^{\left.j\left(\omega_{c}+n \omega_{R F}\right) t+\frac{n \pi}{2}\right)}\right]
\end{gathered}
$$

Where is the optical power misfortune inside the MZM, $\mathrm{m}$ the $R F$ regulation list characterized as $m=(V R F v \pi R F)$, $\operatorname{Jn}(\mathrm{m})$ is the nth-request Bessel work, $v \pi R F$ is the exchanging tweak voltage of the MZM.It is obvious from Equation (4) that the odd-request sidebands are altogether stifled as a result of $\cos (\mathrm{n} / 2)$. So the $2 \mathrm{kth}$-request sidebands are created at the yield of the modulator, where $\mathrm{k}$ is a whole number. The yield of the first MZM is sent as contribution to the second MZM since both are associated in course. The RF-driven sign applied to second MZM

$$
\begin{aligned}
& v_{a}(t)=V_{R F} \sin \left(\omega_{R F} t+\Delta \emptyset_{1}\right) \quad v_{b}(t)=V_{R F} \\
& \sin \left(\omega_{R F} t+\Delta \emptyset_{1}+\Delta \emptyset_{2}\right)
\end{aligned}
$$

Where the stage move $\Delta \varnothing 1=90^{\circ}$ is presented between the RF driving sign on the MZMs, and the stage move $\Delta \varnothing 2=180^{\circ}$ is presented between the RF driving sign on the arms of every one of the MZMs. The last yield toward the finish of the second MZM can given as

$$
\begin{aligned}
& E_{02}(t)=\frac{\alpha^{2}}{2} E_{c} \\
& {\left[\sum_{n=-\infty}^{\infty} J_{n}(m) \cos \cos \left(\frac{n \pi}{2}\right) e^{j\left(\left(\omega_{c}+n \omega_{R F}\right) t+\frac{n \pi}{2}\right)}\right]} \\
& {\left[e^{\left(\frac{j \pi v_{b}(t)}{v_{\pi R F}}\right)}+e^{\left(j \pi v_{a}(t) / v_{\pi R F)}\right.}\right](5)} \\
& \begin{array}{c}
=\frac{\alpha^{2}}{2} E_{c} \\
{\left[\sum_{n=-\infty}^{\infty} J_{n}(m) \cos \cos \left(\frac{n \pi}{2}\right) e^{j\left(\left(\omega_{c}+n \omega_{R F}\right) t+\frac{n \pi}{2}\right)}\right]}
\end{array} \\
& {\left[e^{\left(\frac{-j \pi V_{R F} \cos \left(\omega_{R F} t\right)}{v_{\pi R F}}\right)}+\quad e^{\left(j \pi V_{R F} \frac{\cos \left(\omega_{R F} t\right)}{v_{\pi R F}}\right)}\right]} \\
& =\alpha^{2} E_{c} \\
& {\left[\sum_{n=-\infty}^{\infty} J_{n}(m) \cos \cos \left(\frac{n \pi}{2}\right) e^{j\left(\left(\omega_{c}+n \omega_{R F}\right) t+\frac{n \pi}{2}\right)}\right]} \\
& {\left[\sum_{n=-\infty}^{\infty} J_{n}(m) j^{n} \cos \left(\frac{n \pi}{2}\right) e^{j\left(\left(n \omega_{R F}\right) t+\frac{n \pi}{2}\right)}\right]} \\
& \left.=\alpha^{2} E_{c} \sum_{n=-\infty}^{\infty} J_{n}^{2}(m) j^{n}\left(\frac{n \pi}{2}\right) e^{j\left(\left(\omega_{c}+2 n \omega_{R F}\right) t+n \pi\right)}\right] \\
& E_{02}(t)=\alpha^{2} E_{c}\left\{J_{2}^{2}(3.39)\right\} \\
& {\left[-e^{j\left(\left(\omega_{c}+4 \omega_{R F}\right) t\right)}+\right.} \\
& \left.e^{j\left(\left(\omega_{c}-4 \omega_{R F}\right) t\right)}\right] \quad(6)
\end{aligned}
$$

The zeroth-request Bessel work gets zero during the condition $\mathrm{m}=3.39$. In this condition the optical bearer is stifled. It tends to be found in Equation (6), that the fourthrequest sidebands are acquired and the others are suppressed.Notably, no optical channel is required to expel undesired optical sidebands.The electrical sign with recurrence multiple times that of the RF driving sign is gotten. 


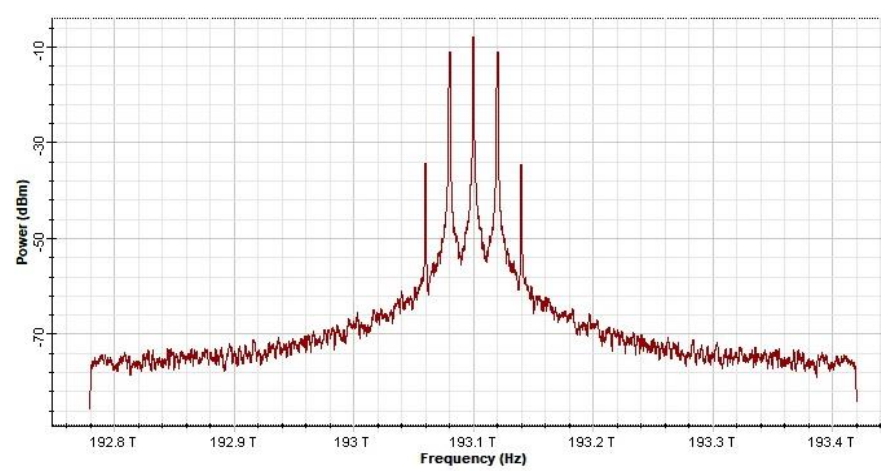

Figure 2. Optical spectrum of the signal generated from the first stage of the system.

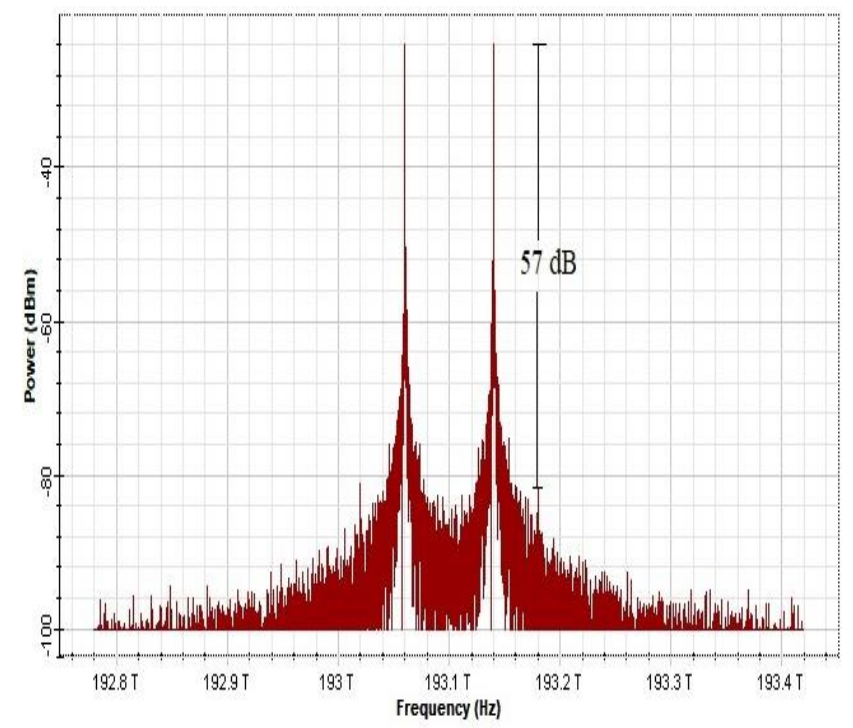

Figure 3. Optical spectrum of the generated signal from the frequency octupling system.

\section{RESULTS AND DISCUSSION}

Following the setup shown in Figure.1, a continuous-wave (CW) laser with a wavelength of $1554 \mathrm{~nm}$ is used as the optical source and it is sent to first MZM of the system.To generate $80-\mathrm{GHz}$ MMW signal, a 10-GHz sinusoidal driving sign is used.The two MZMs are driven by a similar RF sign of $10 \mathrm{GHz}$ with a stage contrast of $90^{\circ}$ introduced by an

electrical stage shifter.Figure 2, shows the optical spectrumof the MMW signal produced at the yield of the main phase of the framework. Also, it unmistakably shows just the even request sidebands with the bearer. The intensity of the second request sideband is $-10 \mathrm{dBm}$ and is recognizably higher than the other sidebands. The tweak lists of both MZMs are about 3.39. Figure 3, shows the optical range of the MMW signal created at the yield of the framework with recurrence octupling system. It tends to be seen that the created optical MMW fundamentally has two tones (fourth-request sidebands) with a recurrence dispersing of $80 \mathrm{GHz}$ and the optical bearer and other undesired optical sidebands are stifled well.And it is likewise observed that the intensity of the fourth-request sideband is $-24 \mathrm{dBm}$ and it is seen as perceptibly high. It is seen from Figure 4 that the RF range principally comprises of the ideal $80 \mathrm{GHz}$ MMW signal and the RFSSR is seen to be $42 \mathrm{~dB}$. Figure 2 and 3 shows that the recreation results concur with the hypothetical investigation in Section 2 ,
Equations (4) and (6). After the recurrence octupling framework, an EDFA is used to help the optical power.

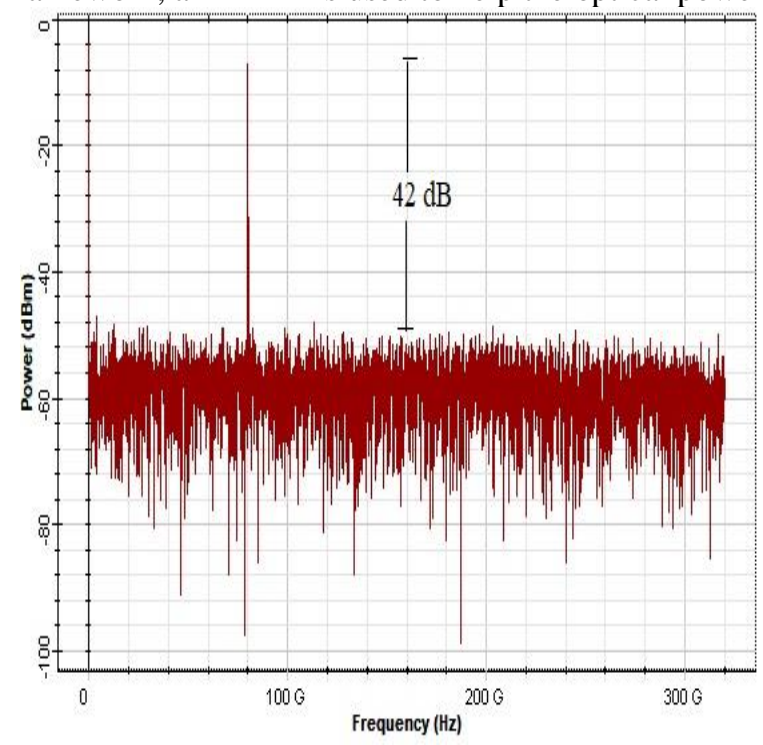

Figure 4. RF spectrum of the generated signal.

The effect of non-perfect drive voltage of RF-driven sign on OSSR and RFSSR is appeared in Figure 5. The variety of the OSSR and RFSSR versus the deviation of the determined voltage is seen as indistinguishable. The OSSR more prominent than $55 \mathrm{~dB}$ and the RFSSR more noteworthy than $40 \mathrm{~dB}$ must be gotten for a $\mathrm{RF}$ drive voltageof 4.33. Figure 5 shows the debasement of the sideband concealment proportion because of the different $\mathrm{RF}$ driven voltages applied to MZM. It is seen that just at the look estimation of the RF drive voltage at 4.33, the OSSR and RFSSR isfound to have a greatest worth. As the $\mathrm{RF}$ drive voltage diminishes or increments past the estimation of 4.33, there is seen as a significant reduction in the estimations of OSSR and RFSSR. As checked byexperiments, the most extreme estimation of RFSSR is seen as $42 \mathrm{~dB}$ and the greatest estimation of OSSR is seen as $57 \mathrm{~dB}$

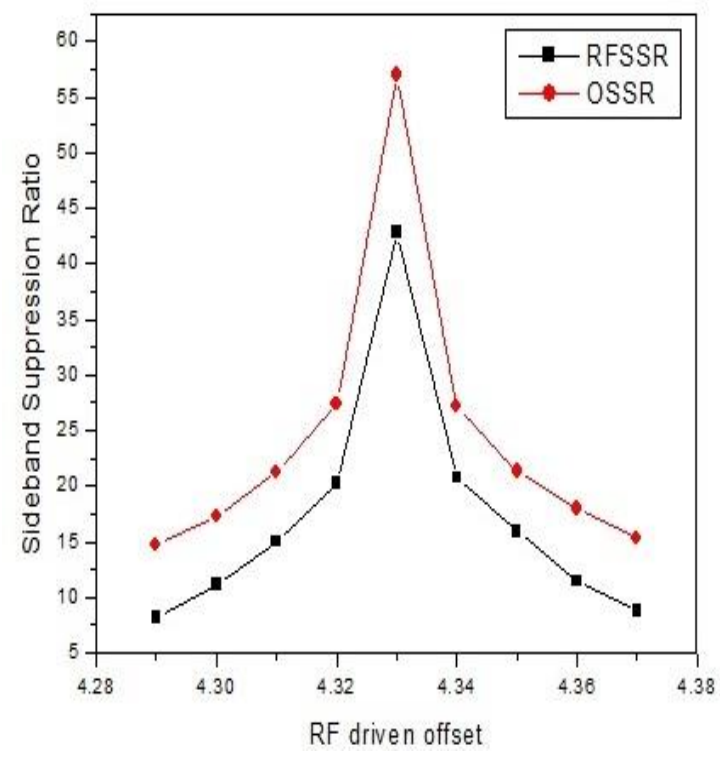

Figure 5.Sideband Suppression Ratio driving voltage deviation

against RF

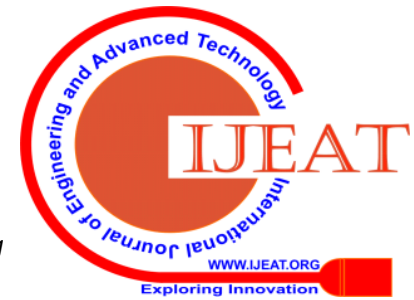


Figure 6 shows the impact of stage move deviation on the Sideband Suppression Ratio. The most elevated OSSR and RFSSR can be gotten for a stage contrast close to the estimation of $90^{\circ}$.The qualities at that point somewhat corrupts with the addition and decrement of deviation esteem for two cases, i.e., RFSSR and OSSR. An OSSR more noteworthy than $55 \mathrm{~dB}$ can be acquired for stage move of $90^{\circ}$, which is an alluring outcome. Figure 6 shows the disintegration of the sideband concealment proportion because of the different stage shifts applied to MZM. The $90^{\circ}$ phase contrast between the driving sign of the first and second MZM is a key factor which influences the concealment of the undesired sidebands. The optical sideband concealment proportion will debase if the stage contrast isn't equivalent to $90^{\circ}$. The effect of the non-perfect voltage of RF-driven sign and the effect of the stage move between the two driving sign is found to have an indistinguishable effect.The best sideband concealment proportion is gotten with a 90-deg stage delay and a RFdrive voltage

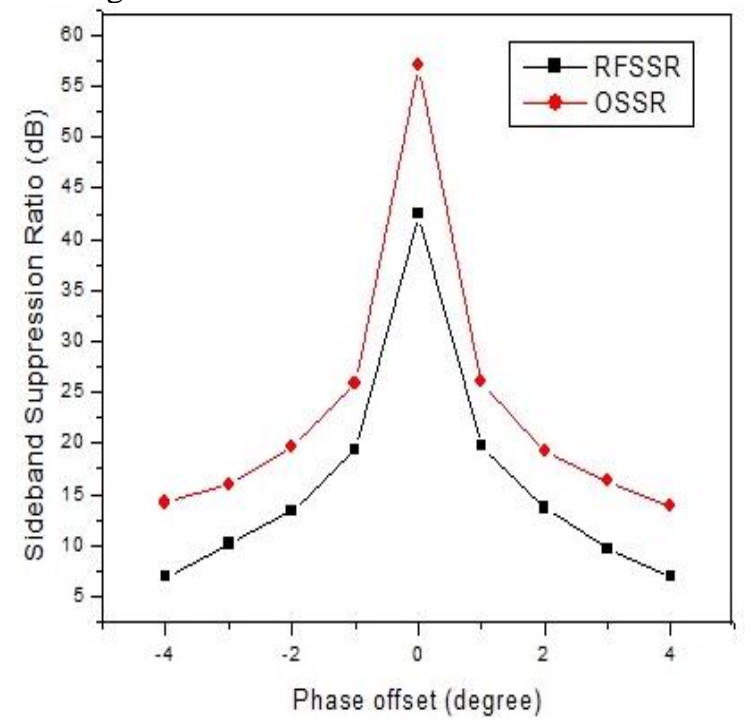

Figure 6.Sideband Suppression Ratio against phase

\section{CONCLUSION}

In this paper, a filterless photonic methods for creating MMW signal with recurrence octupling system has been displayed. It is a practical technique to create a great octupling recurrence optical MMW signal utilizing two fell MZMs . The recurrence octupling plan can additionally diminish the recurrence necessity of the information signal. The proposed plan is tentatively explored and examined .The created optical MMW signal is relied upon to show great transmission execution over a long separation.

\section{REFERENCES}

1. A. J. Seeds K. J. Williams, "Microwave photonics", J. Lightw. Technol. Vol. 24, no. 12, 4628-4641, Dec. 2006.

2. A. Wiberg, P. Perez-Millan, M. V. Andres, P. O. Hedekvist, "Microwave-photonic frequency multiplication utilizing optical fourwave mixing and fiber Bragg gratings", J. Lightw. Technol. Vol. 24, no.1,329-334, Jan. 2006

3. Kumar, A., B. Suthar, V. Kumar, K. S. Singh, and A. Bhargava,"Tunable wavelength demultiplexer for DWDM application using 1-D photonic crystal," Progress In Electromagnetics ResearchLetters, Vol. 33, 27-35, 2012.
4. Par, Vol. 25k, C., C. G. Lee, C. S. Park, "Photonic frequency Up conversion by SBS-based frequency tripling," Journal of LightwaveTechnology, No. 7, 1711-1718, 2007.

5. Wang, Q., H. Rideout, F. Zeng, and J. Yao, "Millimeter-wave frequency tripling based on four-wave mixing in a semiconductor optical amplifier," IEEE Photonics Technology Letters, Vol. 18,No. 23, 2460-2462, 2006.

6. Yu, J., Z. Jia, L. Yi, Y. Su, G. K. Chang, T. and Wang, "Optical millimeter-wave generation or up-conversion using external modulators," IEEE Photonics Technology Letters, Vol. 18, No. 1, 265-267, 2006.

7. Shi, P.Et Al, "A frequency sextupling scheme for high-quality optical millimeter-wave signal generation without optical filter," Optical Fiber Technology, Vol. 17, 236-241, 2011.

8. N. A. Al-Shareefi, S. I. S. Hassan, M. F. B. A. Malek, R. Ngah, S. A. Abbas, and S. A. Aljunid, "A cost- effective method for high-quality $60 \mathrm{GHZ}$ optical millimetre wave signal generation based on frequency quadrupling", Progress In Electromagnetics Research, Vol. 137, 255274, 2013

9. Chun-Ting Lin, Yu-Min Lin, Jason (Jyehong) Chen, Sheng-Peng Dai, Po Tsung Shih, Peng-Chun Peng, and Sien Chi," Optical directdetection OFDM signal generation for radio-over-fiber link using frequency doubling scheme with carrier suppression"OSA Publishing , Optics Express, Volume 16 ,Issue 9,2008

10. G. Qi J. P. Yao J. Seregelyi C. Belisle S. Paquet, "Generation and distribution of a wide-band continuously tunable millimeter-wave signal with an optical external modulation technique" IEEE Trans. Microw. Theory Tech., Vol. 53 no. 10 pp. 3090-3097 Oct. 2005.

11. J. Zhang H. Chen M. Chen T. Wang S. Xie "A photonic microwave frequency quadrupler using two cascaded intensity modulators with repetitious optical carrier suppression",IEEE Photon. Technol. Lett., Vol. 19, no. 14, 1057-1059 Jul. 2007

12. M. Mohamed X. Zhang B. Hraimel K. Wu, "Frequency sixupler for millimeter-wave over fiber systems" Opt. Express, Vol. 16, no. 14, 10141-10151, Jun. 2008.

13. J. Zhang H. Chen M. Chen T. Wang S. Xie,"Photonic generation of a millimeter-wave signal based on sextuple frequency multiplication" Opt. Lett., Vol. 32, no. 9, 1020-1022, Apr. 2007.

\section{AUTHORS PROFILE}

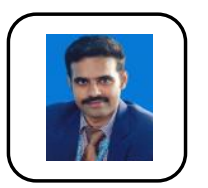

K. Vijayakumar completed his Bachelor's Degree in Computer Science and Engineering from Anna University, Tamil Nadu, India. He received his Master's Degree \& Doctoral Degree in Information Technology from Sathyabama University, Tamil Nadu, India. He has 13 years of teaching experience and 6 years of research experience so far. He is presently working as an Associate Professor at St. Joseph's Institute of Technology, Chennai, India. His area of specialization includes Data Mining, Cloud Computing, Risk Assessment, Machine Learning and Network Security. He has published more than 20 research papers in International Journals and has presented 10 Papers in International Conferences. His works have been cited in more than 100 research articles with i10-index 4 and h-index 5 . He has edited over 400 papers in various reputed journals $\mathrm{He}$ is also a reviewer of more than 30 Journals including Sage, Springer, Elsevier, Inderscience publishers, etc. $\mathrm{He}$ is an editorial board member of more than 20 reputed International journals. He has published a book titled "Computer Programming" ISBN13: 978-93-5260-311-4, ISBN-10: 93-5260-311-7 published by McGraw Hill Education Pvt. Ltd., 2016.

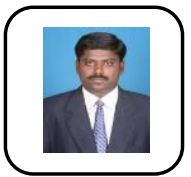

Baskaran.m is an associate professor at the department of electronics and communication engineering, st. Joseph's institute of technology, chennai, india. He received the m.e. degree in anna university chennai, india, in 2005 . He is currently working toward the ph.d. Degree at anna university chennai, india. His research interests include radio over fiber technologies, microwave photonics and optical communication. 